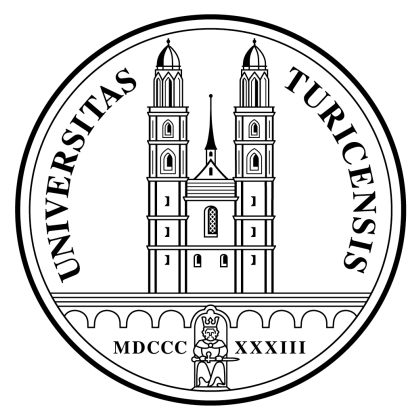

Institute for Empirical Research in Economics

University of Zurich

Working Paper Series

ISSN 1424-0459

Working Paper No. 182

Heterogeneity, Local Information, and Global Interaction

Aleksander Berentsen, Esther Bruegger, Simon Loertscher

February 2004 


\title{
Heterogeneity, Local Information, and Global Interaction*
}

\author{
Aleksander Berentsen, Esther Bruegger, Simon Loertscher ${ }^{\dagger}$ \\ February 5, 2004
}

\begin{abstract}
Consider a society where all agents initially play "fair" and one agent invents a "cheating" strategy such as doping in sports. Which factors determine the success of the new cheating strategy? In order to study this question we consider an evolutionary game with heterogenous agents who can either play fair or cheat. We model heterogeneity by assuming that the players are either high or low types. Three factors determine the imitation dynamics of the model: the location and the type of the innovator, the distribution of types, and the information available to the agents. In particular we find that the economy is more likely to end up in a state where all agents cheat if the innovator is of low type or when the agents are maximally segregated.
\end{abstract}

Keywords: Evolutionary game theory, imitation dynamics, heterogeneity, local information, global interaction.

JEL-Classification: C70, C79, D83.

*This paper has benefited from the comments of seminar participants at the University of Bern and the Max Planck Institute for Research into Economic Systems in Jena. We also thank Cédric Wasser and Yvan Lengwiler for their valuable comments, Kurt Schmidheiny for assisting the programming of the MATLAB code, and Andres Aeschliman for providing us access to the large-capacity computer for our simulations. The second and third author gratefully acknowledge financial support received from the WWZ-Forum Basel (B-61).

$\dagger$ Berentsen: Economics Department, University of Basel, Petersgraben 51, CH-4003 Basel. E-mail: aleksander.berentsen@unibas.ch. Brügger: Economics Department, University of Bern, Gesellschaftsstrasse 49, CH-3012 Bern. E-mail: esther.bruegger@vwi.unibe.ch. Lörtscher: Economics Department, University of Bern, Vereinsweg 23, CH-3012 Bern. E-mail: simon.loertscher@vwi.unibe.ch. 


\section{Introduction}

In this paper we consider an evolutionary game where agents competing for a prize can either play fair or cheat. We want to identify the determinants that eventually lead to an absorbing state of the imitation dynamics in which all agents use the cheating strategy when initially all but one agent play fair. We are interested to identify the role of heterogeneity and the role of information for this process. In particular, we would like to know how the distribution of types, i.e. the segregation of the population determines the imitation dynamics.

To study these issues we consider an evolutionary game in which a finite number of infinitely lived agents are matched pairwise to play a $2 \times 2$ stage game. In each stage game, the agents compete for a prize of value $w$ by either playing "fair" or "cheat." Agents are of two types: high or low. High types have a natural advantage over low types: they obtain the prize $w$ with certainty if they meet a low type and if both use the same strategy. If, however, a low type cheats against a fair playing high type, he beats the high type with certainty and receives the prize $w$. If two players of the same type meet and if both use the same strategy, they share the prize of value $w$. Cheating is costly where the cost $c$ satisfies $0<c<w{ }^{1}$

Because our agents are heterogenous there are two stage games. First, if two agents of the same type meet, the stage game is a Prisoner's Dilemma where cheating (playing fair) is the dominant strategy if the cost of cheating is sufficiently low (high). Second, if two agents of opposite type meet, the stage game is an asymmetric game similar to a matching penny game. In this game, the low type's best response is the strategy that is not used by the high type, and the high type's best response is the strategy that the low type is using.

In each period each agent is matched sequentially to all other agents, i.e. agents interact globally. At the end of the period, each agent observes the strategies and average payoffs of a subset of all agents, called the information set of an agent. In the following period, agents imitate the strategy with the highest average payoff in their information set. ${ }^{2}$ To formalize these sets we locate the

\footnotetext{
${ }^{1}$ One application we have in mind is the spread of a new doping substance in sports, where athletes with different talents may learn how to use this substance to improve their winning probabilities. Other applications are crime or corruption. The relevant question here is under which circumstances a previously peaceful (honest) society can turn violent (corrupt).

${ }^{2} \mathrm{~A}$ few years ago at the Tour de France, some cyclists began to use somewhat odd looking
} 
agents on a circle as in Ellison (1993). In most parts of the paper, we focus on two information settings: "local" information and "global" information. With local information agents observe the strategies and payoffs of their immediate neighbors on the circle only. If agents have global information, they observe the strategies and payoffs of all agents. ${ }^{3}$

In order to get a benchmark we first analyze the model when all agents are identical. In this case the stage game is a Prisoner's Dilemma where cheating is the dominant strategy if $c<w / 2$. With equal agents we find that the information setting has no influence for the set of absorbing states. That is, we show that the cardinality of the information set only affects the time elapsing until the set of absorbing states is reached. Moreover, the location of the innovator of the cheating strategy is irrelevant - a result which is hardly surprising given that all agents are equal.

These results motivated us to study the role of heterogeneity in our model. With heterogenous agents there are three crucial factors that determine the spread of the cheating strategy: the location and the type of the innovator of the cheating strategy, the distribution of types on the circle, and the information available to the agents. These factors determine whether the innovator is able to infect his neighbors and eventually to contaminate the entire population. Since many different distributions of types on the circle are feasible - each of them having potentially different implications for the limiting distribution - we focus on two polar cases: maximal segregation and minimal segregation. In a maximally segregated population high types and low types are allocated in two clusters so

nose stickers. They were supposed to increase breathing capacity and thereby the prospects of winning the race. Very soon after the first few cyclists had used these stickers, they were adopted by virtually all others. However, the following year, the stickers had almost disappeared again presumably because they did not enhance performance. This is an example of the imitation behavior we have in mind. We belief that it also describe well how illegal performance enhancing substances, which are not observed in public, spread in sports.

${ }^{3}$ In sports, say tennis, all agents compete against all other agents (global interaction). Nevertheless, the players - for obvious reasons - only share information about the use of illegal performance enhancing drugs with their best mates (local information). To describe a situation in which agents interact globally but have only local information about payoffs and strategies, consider a finite number of myopic agents in a computer lab where the computers are allocated in a circle. In each period each agent plays a stage game against each other agent in the lab. At the end of the period each agent observes his average payoff for the period. In addition he observes the screens of his immediate neighbors which reveals their strategies and their average payoffs in that period. Using this information, each agent at the end of the period imitates the strategy with the highest average payoff. 
that there are only two players of each type that have a neighbor of the opposite type. In a minimally segregated population each agent has two neighbors of opposite type.

The following results emerge from the model with heterogenous agents and local information. First, the population is more likely to end up in an absorbing state where all agents cheat if the innovator is a low type. Second, if the innovator is a low type, then a minimally segregated population is more resistant against the cheating strategy than a maximally segregated population. The reason for this result is that in a minimally segregated population each low type (including the innovator) is surrounded by high types who are less prone to imitate the cheating strategy. Third, in contrast, if the innovator is a high type, a maximally segregated population is more resistant against the cheating strategy than a minimally segregated population. The reason for this result is that the innovator is again surrounded by high types who are less prone to imitate the cheating strategy.

The role of information with heterogenous agents is as follows. First, with global information, in contrast to local information, the location and the type of the innovator of the cheating strategy and the distribution of types on the circle are irrelevant. Second, local information reduces the spread of the cheating strategy if agents are minimally segregated relative to a situation where agents have global information. For a maximally segregated population this result is only true if the innovator is a high type. Third, with local information some agents under- and some overestimate the true benefit of the cheating strategy. There is no such effect with complete information where each player type knows the true benefit of each strategy for his type.

Our paper is most closely related to Ellison (1993), Eshel et al. (1998), and Kandori et al. (1993). Kandori et al. (1993) consider the limiting distribution when individual mutation rates go to zero for the class of $2 \times 2$ stage games. The players' period payoffs are the expected values of the stage game given the (distribution of) strategy choices of all players. Like Kandori et al. (1993) we assume "global interaction." Ellison (1993) investigates the limiting distributions and the speed of convergence in a similar model as Kandori et al. (1993). The crucial difference is that players interact and obtain information locally. Also, Ellison (1993) focuses on coordination games. 
Summarizing, Kandori et al. (1993) investigate global interaction and global information and Ellison (1993) local interaction and local information. In contrast, we combine local information with global interaction. Moreover, we introduce heterogeneity among agents, which generates different stage games. Finally, we adopt the imitation rule of Eshel et al. (1998) where players can only play the strategies they observe in their information set. Like Ellison (1993), Eshel et al. (1998) may be classified as a local interaction and local information game.

The paper is organized as follows. In Section 2, we describe the basic model with homogenous agents. Sections 3 and 4 analyze the model with heterogenous agents and global and local information, respectively. In Section 5, we allow for mutations. Section 6 concludes. 


\section{The Model with Homogeneous Agents}

In this section, we set up the model with homogeneous agents. We first describe the stage game, specify how agents are located and how they adopt or choose new strategies. We then analyze the absorbing strategy states.

\subsection{The Stage Game}

We consider a finite population with $N>1$ agents denoted by $i=1, \ldots, N$ who live forever. In every period $t$, the agents are sequentially matched to all other agents to play a $2 \times 2$ stage game. In each stage game, the agents compete for a prize of value $w$. The strategy space is $\{C, D\}$, where $C$ stands for playing "clean" respectively fair and $D$ for cheating respectively playing "doped." Within a period, a player cannot change his or her pure strategy. Furthermore, mixed strategies are ruled out.

The payoffs of the stage game are as follows. If both agents play the strategy $C$, each gets $\frac{w}{2}$. If both play $D$, each gets $\frac{w}{2}-c$, where $c \in(0, w)$ is the cost of cheating. It reflects the fact that each agent prefers to obtain the prize $w$ by playing $C$ rather than by using $D$. Finally, if an agent plays $D$ against a clean player, he gets $w-c$ and the clean player 0 . Thus, the payoff matrix is

$$
A=\left(\begin{array}{cc}
\frac{w}{2} & 0 \\
w-c & \frac{w}{2}-c
\end{array}\right) \text {. }
$$

The stage game defined in (1) is a Prisoner's Dilemma (Weibull 1995). If $c \lessgtr \frac{w}{2}$, $D$, respectively $C$, is the dominant strategy.

Agent $i$ 's period payoff in period $t, u_{i, t}$, is the average payoff from the $N-1$ matches, i.e.

$$
u_{i, t}\left(\sigma_{i, t}, \sigma_{-i, t}\right)=\frac{1}{N-1} \sum_{-i} a\left(\sigma_{i, t}, \sigma_{-i, t}\right)
$$

where $\sigma_{i, t}$ is his strategy in period $t, \sigma_{-i, t}$ are the strategies chosen by all other players in period $t$, and the payoffs $a(.,$.$) are the corresponding elements of A$ in (1).

\subsection{Location and Imitation}

In order to model incomplete information, we assume that agents are located on a circle on the positions $1,2,3, \ldots, N$. In each period, each agent $i$ obtains 
information about the period payoffs and the strategies chosen by the agents $i \pm k$ (modulo $N$ ) with $k \in\left\{1, \ldots,\left\lfloor\frac{N}{2}\right\rfloor\right\}$, where $\left\lfloor\frac{N}{2}\right\rfloor$ is the largest integer $\leq \frac{N}{2}$. Dropping the arguments in $u_{i, t}\left(\sigma_{i, t}, \sigma_{-i, t}\right)$, we define agent $i$ 's information set $G_{i, t}(k)$ as

$$
G_{i, t}(k)=\left\{\left(u_{j, t}, \sigma_{j, t}\right) \mid j=i-k, \ldots, i+k\right\} .
$$

If $k=\left\lfloor\frac{N}{2}\right\rfloor$, the information set contains information about all agents on the circle. In this case, we say that agents have global information. If $k<\left\lfloor\frac{N}{2}\right\rfloor$ the information set contains not all relevant information. If $k=1$, agents observe strategies and payoffs of their direct neighbors only. We call this information setting local information.

We now turn to the question how agents use their information. Following Eshel et al. (1998), we assume that at the end of every period $t$, the agents observe $G_{i, t}(k)$. In the following period, they play the strategy that generated the highest average payoff. ${ }^{4}$ If they observe but one strategy within their information set, they play this strategy next period. Let $d_{j, t}$ denote an indicator variable, which takes the value 0 if agent $j$ plays $C$ and the value 1 if he plays $D$. Then, if strategies $C$ and $D$ are observed in $G_{i, t}(k)$, the observed payoff difference $\Delta_{i, t}$ is

$$
\Delta_{i, t}=\frac{\sum_{j=i-k}^{i+k} d_{j, t} \cdot u_{j, t}}{\sum_{j=i-k}^{i+k} d_{j, t}}-\frac{\sum_{j=i-k}^{i+k}\left(1-d_{j, t}\right) \cdot u_{j, t}}{\sum_{j=i-k}^{i+k}\left(1-d_{j, t}\right)} .
$$

The first term is the average payoff of those agents in the information set that play $D$ and the second term is the average payoff of those agents that play $C$. The imitation dynamics satisfies the following rule.

Definition 1 The imitation rule is

$$
\sigma_{i, t+1}=\left\{\begin{array}{ccc}
\sigma_{i, t} & \text { if } & \Delta_{i, t}=0 \\
C & \text { if } & \Delta_{i, t}<0 \\
D & \text { if } & \Delta_{i, t}>0
\end{array} .\right.
$$

if both strategies $C$ and $D$ are observed in $G_{i, t}(k)$. Otherwise, the agent continues to use the same strategy.

\footnotetext{
${ }^{4}$ Agents who choose strategies according to an imitation rule can interpret the information they receive in two different ways. They can either imitate the most successful player or the most successful strategy they observe. The former imitation rule is used by Vega-Redondo (1997) and Alos-Ferrer et al. (2000) and others, the latter e.g. by Ellison and Fudenberg (1995). We will adhere to an imitation rule of the second kind, where success of a strategy is measured by its average payoff.
} 
The imitation rule implies that if $\Delta_{i, t}=0$, then $\sigma_{i, t+1}=\sigma_{i, t}$, and for $\Delta_{i, t} \lessgtr 0$, we have $\sigma_{i, t+1}=C$, respectively $D$. If a strategy is not observed in an agent's information set, then the agent continues to use the strategy of the current period. Note that an agent's behavior depends solely on the strategies observed in his neighborhood in the immediate past. That is, neither the shadow of the future nor the shadow of the more distant past bear any weight for the choice of strategy (Berninghaus et al. 2003).

In the following we will suppress the time index $t$. We denote by $y$ the number of agents playing $C$. This allows us to write agent $i$ 's payoff of playing $d_{i} \in\{0,1\}$ as

$$
u_{i}\left(d_{i}, y\right)=\frac{y-1}{N-1} \frac{w}{2}+d_{i}\left(\frac{N}{N-1} \frac{w}{2}-c\right) .
$$

The first term in (3) is the period payoff of an agent who plays $C\left(d_{i}=0\right)$. The second term, which depends only on the population size $N$ and the cost $c$, is the additional payoff for an agent of playing $D\left(d_{i}=1\right)$. For a homogeneous population, (3) implies that an agent $i$ who observes $C$ and $D$ in $G_{i}(k)$ compares $u_{j}(1, y)$ and $u_{h}(0, y)$ for some $j, h$. Thus, $\Delta_{i}=u_{j}(1, y)-u_{h}(0, y)=\frac{N}{(N-1)} \frac{w}{2}-c$. Note that $\Delta_{i}$ is independent of $y$.

The model exhibits a finite population effect. Because agents do not play against themselves, $\Delta_{i}$ only approaches the value $\frac{w}{2}-c$ as $N$ goes to infinity. Thus, for any finite $N$ and for $c \in\left(\frac{w}{2}, \frac{N}{N-1} \frac{w}{2}\right), D$ is a strictly dominated strategy and nevertheless $\Delta_{i}>0$ for all $i$ who observe the strategy $D$ in their neighborhood. That is, despite $D$ being a strictly dominated strategy, all individuals will end up playing it. The reason for this result is that an agent playing $D$ is matched to one $D$-player less than an agent playing $C$. This increases the benefit of using strategy $D$ relative to $C$.

\subsection{Absorbing States}

A state is a specification of which agents play $C$ and which play $D$. At time $t$, we describe the state $s_{t}$ of the system by an $N$-tuple

$$
s_{t}=\left(\sigma_{1 t}, \sigma_{2 t}, \ldots, \sigma_{N t}\right) \in S \equiv\{C, D\}^{N},
$$

where $S$ is the set of possible states. If $i$ and $j$ are two possible strategy states in $S, p_{i j}$ is the probability that the imitation rule changes the system to state $j$ 
given that $i$ is the current state. The imitation rule in (2) and the non-stochastic nature of the payoffs result in a deterministic process such that $p_{i j}$ is either 0 or 1. The collection $\left\{p_{i j}\right\}_{i, j \in S}$, together with an initial state, is a Markov process on $S$. We will refer to this Markov process as the imitation dynamics of our model.

We are interested in the absorbing states of the imitation dynamics, which are defined as in Eshel et al. (1998).

Definition 2 A set of states is absorbing if it is a minimal set of states with the property that the Markov process can lead into this set but not out of it.

An absorbing set of states may contain only one state. If an absorbing set contains more than one state, the Markov process cycles between the states contained in the absorbing set.

¿From now on, we normalize $w=1$ (and consequently $c$ is now assumed to be $\in(0,1)$ ). Moreover, we concentrate on the polar cases; either the size of the information set is $k=1$ or $k=\left\lfloor\frac{N}{2}\right\rfloor$. In the case of local information $(k=1)$, each agent observes the strategies and the payoffs of his direct neighbors only. In the case of global information $\left(k=\left\lfloor\frac{N}{2}\right\rfloor\right)$, each agent obtains information about the strategies and the payoffs of all agents on the circle.

\subsection{The Role of Information}

Throughout the paper, we study the spread of $D$ in a population in which in $t=1$ all agents but one play $C$.

\subsubsection{Global Information}

If $\sigma_{i}=C(D) \forall i$, we denote this state by $\vec{C}(\vec{D})$. Under global information, we obtain the following result.

Proposition 1 When agents are homogenous and have global information, then the following is true. If $c \lessgtr \frac{N}{2(N-1)}$, the absorbing state $\vec{D}$, respectively $\vec{C}$, is reached in period 2 .

The proof is straightforward. If all agents observe the strategies and payoffs of all other agents, $\Delta_{i}$ is the same for all $i=1,2, \ldots, N$. Consequently, when an agent introduces $D$ in period 1 , depending on whether $\Delta_{i}$ is positive or negative, all agents will play $C$ or $D$ from period 2 on until the end of time. This result has been shown to hold by Kandori et al. (1993) in a more general setting. 


\subsubsection{Local information}

We now consider the case where the agents observe their immediate neighbors only $(k=1)$.

Proposition 2 When agents are homogenous and have local information, then the following is true. If $c<\frac{N}{2(N-1)}$, the absorbing state $\vec{D}$ is reached in period $t=1+\left\lfloor\frac{N}{2}\right\rfloor$. If $c>\frac{N}{2(N-1)}$, the absorbing state $\vec{C}$ is reached in period 2 .

The proof of Proposition 2 is intuitive. If $c<\frac{N}{2(N-1)}, \Delta_{i}$ is positive and all agents that are aware of strategy $D$ imitate it in the following period. Because $k=1$, it takes $\left\lfloor\frac{N}{2}\right\rfloor$ periods until all agents have learned and adopted $D$. If $c>\frac{N}{2(N-1)}, \Delta_{i}$ is negative. Consequently, $D$ dies out immediately.

Comparing Proposition 1 with Proposition 2, we see that the size of the information set only affects the time elapsing until the absorbing state is reached. In particular, it does not matter which player introduces strategy $D$ because all agents are identical. In the following section we therefore introduce heterogeneity among the agents to see how this influences the imitation dynamics.

\section{Heterogenous Agents with Global Informa- tion}

In this section we investigate the role of heterogeneity when agents are globally informed. In Section 4 we then analyze local information.

\subsection{Asymmetric Games}

We introduce heterogeneity by assuming that agents are either of high type $(H)$ or low type $(L)$. An agent's type is neither known by himself nor by any other agent. Agents are still matched pairwise and the payoffs are as follows. If an $H$-type is matched to an $L$-type and both agents use the same strategy (either $D$ or $C$ ), the $H$-type wins with certainty. If an $H$-type is matched to an $L$-type and only the $L$-type uses $D$, then the $L$-type wins with certainty. Thus, an $L$-type prevails over an $H$-type if and only if he plays $D$ and the latter plays $C$. The payoff matrices for the asymmetric matches are

$$
A_{H, L}=\left(\begin{array}{cc}
w & 0 \\
w-c & w-c
\end{array}\right) \text { and } A_{L, H}=\left(\begin{array}{cc}
0 & 0 \\
w-c & -c
\end{array}\right)
$$


where for example $A_{H, L}$ denotes the payoffs to agent of type $H$ when playing against an agent of type $L$. For symmetric matches, the matrices are $A_{H, H}=$ $A_{L, L}=A$ as in (1).

If two agents of the same type meet, the stage game is a prisoner's dilemma where strategy $D$ is the dominant strategy if $c<1 / 2$ as with homogenous agents. In contrast, if two agents of opposite type meet the game is asymmetric and has a unique Nash equilibrium in mixed strategies. In this case, the stage game is a matching penny game where each player's best response is the strategy not chosen by the other agent.

We denote by $y_{H}$ the number of $H$-types playing $C$, and by $y_{L}$ the number of $L$-types playing $C$. The numbers of $H$-types and $L$-types are denoted by $n_{H}$ and $n_{L}$, respectively, with $n_{H}+n_{L}=N$. The period payoffs of $H$-types and $L$-types are

$$
\begin{aligned}
& u_{H}\left(d_{i}, y_{H}, y_{L}\right)=\frac{y_{H}+2 y_{L}-1}{2\left(n_{H}+n_{L}-1\right)}+d_{i}\left(\frac{n_{H}+2 n_{L}-2 y_{L}}{2\left(n_{H}+n_{L}-1\right)}-c\right) \text { and } \\
& u_{L}\left(d_{i}, y_{H}, y_{L}\right)=\frac{y_{L}-1}{2\left(n_{H}+n_{L}-1\right)}+d_{i}\left(\frac{n_{L}+2 y_{H}}{2\left(n_{H}+n_{L}-1\right)}-c\right) .
\end{aligned}
$$

Note that the second term in (4) depends negatively on $y_{L}$, while the second term in (5) depends positively on $y_{H}$. The additional value of playing $D$ for $H$-types (i.e. the second term in (4)) decreases with the number of $L$-types playing $C$. The additional value of playing $D$ for $L$-types increases with the number of $H$ types playing $C$. This is quite intuitive. Since an $H$-type prevails over an $L$-type playing $C$ with certainty, playing $D$ becomes less attractive the more $L$-types play $C$. On the other hand, because an $L$-type prevails over an $H$-type if and only if he plays $D$ and the $H$-type $C, D$ becomes more attractive to $L$-types as the number of $H$-types playing $C$ increases.

\subsection{The Role of Information}

The imitation rule (2) still applies and we continue to study the spread of $D$ from an initial situation where all agents but one play $C$. A state where all agents of the same type play the same strategy is denoted by $\overrightarrow{\sigma_{H}} \overrightarrow{\sigma_{L}}$ where the first component means that all $H$-types play $\sigma_{H}$, the second component that all $L$-types play $\sigma_{L}$. 
Proposition 3 When agents are heterogenous and have global information, then if $c \lessgtr \frac{n_{H}+n_{L}}{2\left(n_{H}+n_{L}-1\right)}$, the absorbing state $\vec{D} \vec{D}(\vec{C} \vec{C})$ is reached in period 2 .

Proof: The proof is in the Appendix.

According to Proposition 3 when information is global, then it is immaterial which type of player innovates $D$. Intuitively, with global information all agents have the same information. Consequently, all agents follow the same decision rule.

¿From Propositions 2 and 3 we see that heterogeneity does not affect the absorbing states, if agents have information about strategies and payoffs of all agents but no information about types. In the following we will see that heterogeneity matters if agents can recognize the types of all agents, or if they are locally informed about strategies and payoffs. Before we consider local information in Section 4, let us consider the model when all agents observe all strategies, all payoffs, and all types. We call this information structure the "complete information" benchmark. With complete information, agents of the same type make the same strategy decisions. Note that for the complete information benchmark we interpret the imitation rule (2) as follows. When applying (2), agents of the same type compare only payoffs and strategies across agents of their own type. ${ }^{5}$ We obtain the following result.

Proposition 4 When agents are heterogenous and have complete information, then the following is true. If $c<\min \left\{\frac{n_{H}}{2(N-1)}, \frac{n_{L}}{2(N-1)}\right\}$, the absorbing state is $\vec{D} \vec{D}$. If

$$
c \in\left[\min \left\{\frac{n_{H}}{2(N-1)}, \frac{n_{L}}{2(N-1)}\right\}, \max \left\{\frac{n_{H}+2 n_{L}}{2(N-1)}, \frac{2 n_{H}+n_{L}}{2(N-1)}\right\}\right],
$$

the absorbing set is $\{\vec{C} \vec{D}, \vec{D} \vec{D}, \vec{D} \vec{C}, \vec{C} \vec{C}\}$. If $c>\max \left\{\frac{n_{H}+2 n_{L}}{2(N-1)}, \frac{2 n_{H}+n_{L}}{2(N-1)}\right\}$, the absorbing state is $\vec{C} \vec{C}$.

Proof: The proof is in the Appendix.

Since all players have both strategies available at any point in time, agents of the same type will always play the same strategy. Obviously, if costs are small

\footnotetext{
${ }^{5}$ If no agent of one type plays $D(C)$, we assume that agents of this type can calculate the hypothetical payoff of playing $D(C)$ for their type and have both strategies at their disposal at any point in time. Otherwise, $D$ would trivially at most spread among the types where innovation occurred.
} 
(large), all agents play $D(C)$. However, in contrast to the game with global information, there is an absorbing set in which agents cycle between $C$ and $D$. This absorbing set is attained if costs are such that $D(C)$ pays for a single agent of either type when all others play $C(D) .^{6}$

In the remainder of the paper, we concentrate on heterogenous agents with local information.

\section{Heterogenous Agents with Local Information}

With heterogenous types and local information, the allocation of types along the circle matters because it affects the payoffs of the strategies $C$ and $D$ which an agent observes in his information set $G_{i}(1)$. Consequently, in contrast to the case with homogeneous agents, $G_{i}(1)$ does not reveal the true benefit of a strategy to a player. For example, a large payoff of a neighbor can now be due to either the strategy chosen (which is observed) or the unobservable $H$-type. In this section, we first classify the agents according to their location, which determines the perceived period payoff of the strategies $C$ and $D$. We then investigate the implication of local information and heterogeneity for the imitation dynamics.

\subsection{The Role of Information}

An agent whose immediate neighbors are of the same type is called an interior player. We denote interior players by $H^{I}$ and $L^{I}$, respectively. If one neighbor is an $H$-type and the other one an $L$-type, we call the player an edge player and denote him by $H^{E}$ or $L^{E}$, respectively. An agent whose two neighbors are not of his type is called a double-edge player, which we abbreviate by $H^{E E}$ and $L^{E E}$, respectively. For the purpose of clarification, we display an example:

$$
H^{E} H^{E} L^{E} L^{I} L^{E} H^{E E} L^{E E} H^{E} H^{I} H^{I} H^{I} H^{E} L^{E} L^{E} H^{E} H^{I} H^{E} L^{E E}
$$

Note that the players at both ends $\left(H^{E}\right.$ on position 1 and $L^{E E}$ on position $N$ on the circle) are immediate neighbors. Let us now determine the strategy choices for the three locations by calculating the decision terms $\Delta$ introduced above.

\footnotetext{
${ }^{6}$ Cycling also occurs in Berentsen and Lengwiler (2004). In this model the authors consider the replicator dynamics in a model with heterogenous agents. The stage game is a Prisoner's Dilemma when two agents of the same type meet or a matching penny game when two agents of opposite type meet.
} 
Interior Player An interior player has only information about agents of his own type. Consequently, an interior player only observes differences in payoffs if different strategies have been played. We write these payoff differences as

$$
\begin{aligned}
\Delta_{H^{I}}\left(y_{H}, y_{L}\right) & =u_{H}\left(1, y_{H}, y_{L}\right)-u_{H}\left(0, y_{H}, y_{L}\right)=\frac{n_{H}+2 n_{L}-2 y_{L}}{2(N-1)}-c \\
\Delta_{L^{I}}\left(y_{H}, y_{L}\right) & =u_{L}\left(1, y_{H}, y_{L}\right)-u_{L}\left(0, y_{H}, y_{L}\right)=\frac{n_{L}+2 y_{H}}{2(N-1)}-c .
\end{aligned}
$$

If $\Delta_{H^{I}} \lessgtr 0$ (or $\Delta_{L^{I}} \lessgtr 0$ ), then $H^{I}\left(L^{I}\right)$ plays $C$, respectively $D$ next period.

Edge Player Let us first consider an edge player of type $H$. Such a player has an $L$-type and an $H$-type as neighbors, i.e. either $L H^{E} H$ or $H H^{E} L$. We concentrate on $L H^{E} H$ because $H H^{E} L$ is analyzed in the same way. An $H^{E}$ player faces eight $\left(=2^{3}\right)$ possible strategy strings in $G_{i, t}(1)$. Two strings are $C C C$ and $D D D$. In this case the agent does not change his strategy. The other six strings are

$$
\underbrace{C D D}_{1^{\text {st }}} \underbrace{C C D C D C}_{2^{\text {nd }}} \underbrace{D D C D C D}_{3^{\text {rd }}} \underbrace{D C C}_{4^{\text {th }}} .
$$

Consider, for example, the first term in (8). It means that " $L$ plays $C, H^{E}$ plays $D$ and $H$ plays $D$." The strategy strings $C C D$ and $C D C$ do not differ with respect to the observed average payoffs. In either case, the $L$-type and one $H$-type play $C$, while the other $H$-type plays $D$. Similarly, the strategy strings $D D C$ and $D C D$ yield also the same average payoffs for $D$ and $C$, respectively.

We can summarize the decisions of $H^{E}$ by considering the observed differences $\Delta_{H^{E}}^{q}$, where the superscript $q$ refers to the rank of the term in (8). In the Appendix we show that the following ranking holds:

$$
\Delta_{H^{E}}^{1} \geq \Delta_{H^{E}}^{2} \geq \Delta_{H^{I}} \geq \Delta_{H^{E}}^{3} \geq \Delta_{H^{E}}^{4}
$$

Thus, if for a clean edge player in a $D C C$ string $\Delta_{H^{E}}^{4}>0$, all edge players and all interior players will play $D$ in the following period.

An edge player of type $L$ has an $H$-type and an $L$-type in his information group. We analyze $H L^{E} L$ because $L L^{E} H$ is analyzed in the same way. The possible strategy strings in the information group of $L^{E}$ are given in (8). We show in the Appendix that

$$
\Delta_{L^{E}}^{4} \geq \Delta_{L^{E}}^{3} \geq \Delta_{L^{I}} \geq \Delta_{L^{E}}^{2} \geq \Delta_{L^{E}}^{1}
$$


Next we show that some agents systematically over- or underestimate the true benefit of the cheating strategy $D$. Recall that an interior player only observes agents of his own type. Consequently, his decision term $\Delta_{L^{I}}$ or $\Delta_{H^{I}}$ reflects the true payoff difference of the two strategies for his type, i.e. an interior player's assessment of a strategy is not distorted by heterogeneity. In contrast, according to (9) and (10), edge players systematically over- or underestimate the payoffs of $C$ or $D$ to their type as defined below.

Definition 3 We say that an agent overestimates (underestimates) the payoff of strategy $D$ for his type if his decision term $\Delta$ is greater (smaller) than the decision term of an interior player of his type.

For example, edge players of type $H$ overestimate $D$ whenever their $L$-type neighbor plays $C$, i.e. $\Delta_{H^{E}}^{1} \geq \Delta_{H^{E}}^{2} \geq \Delta_{H^{I}}$. The reason for this is that an $L$-type player's payoff using $C$ is always zero except when matched to another $L$-type player using $C$ in which case his payoff is $1 / 2$. In contrast, an $H$-type who plays $C$ receives a positive payoff when matched to another $H$-type or to an $L$-type using $C$. Consequently, an edge player of type $H$ underestimates the benefit of strategy $C$, respectively overestimates $D$, when his $L$-type neighbor plays $C$.

There are two crucial features of local information. First, certain agents (edge players and double-edge players) under- or overestimate strategy $D$, respectively, $C$. There is no such effect with complete information where each player type knows the true benefit of each strategy for his type. Second, local information permits some agents (interior players) to observe the true payoff difference of the two strategies for his type as explained above. In contrast, with global information and heterogenous players no agent ever observes the true payoff difference for his type.

Double-Edge Player Finally, let us investigate the behavior of double-edge players. The information group for an $H^{E E}$-type and an $L^{E E}$-type respectively are composed of the following types:

$$
L H^{E E} L \text { and } H L^{E E} H \text {. }
$$

Thus, the relevant strategy strings are

$$
\underbrace{D C D}_{1^{\text {st }}} \underbrace{C C D D C C}_{2^{\text {nd }}} \underbrace{D D C \quad C D D}_{3^{\text {rd }}} \underbrace{C D C}_{4^{\text {th }}} \text {. }
$$


In the Appendix we show that the following ranking holds,

$$
\Delta_{H^{E E}}^{3}>\Delta_{H^{I}}>\Delta_{H^{E E}}^{2} \text { and } \quad \Delta_{L^{E E}}^{2}>\Delta_{L^{I}}>\Delta_{L^{E E}}^{3} .
$$

Like edge players, double-edge players over- or underestimate the payoff of strategy $D$ to their type.

\subsection{Maximal Segregation}

Recall that we want to study how the strategy $D$ evolves in a population in which initially all players but one play $C$. The agent who first plays $D$ is called the innovator. Two factors determine the spread of $D$ : The allocation of types along the circle and the location and type of the innovator.

We now consider a distribution of types that we call maximal segregation. In such a population $H$-types and $L$-types are allocated in two clusters as follows:

$$
H H H \ldots H H L L L \ldots L L L
$$

In a maximally segregated population, there are only two edge players for each type and no double-edge players. In order to simplify the analysis, we assume that the number of $H$-types and $L$-types is the same, i.e. $n_{H}=n_{L}=n=\frac{N}{2}$.

\subsubsection{Innovation and absorbing states}

As explained above, for each type there are three classes of agents; interior, edge and double-edge players. Within the same class agents may choose different strategies because they have different information sets. Consequently, we have to distinguish the location of the innovator. Innovation by an interior player has a different implication for the imitation dynamics than an innovation from an edge player. Moreover, we have also to distinguish among interior players. An innovation through an interior player who is located within other interior players has different consequences than an innovation from an interior player who is located next to an edge player. We call these special interior players next-to-edge players and give them the superscript $N E$, while we still denote all other interior players by superscript $I$.

Proposition 5 If an L-type introduces $D$, then for $c<\frac{2 n}{2(N-1)}$ the absorbing state is $\vec{D} \vec{D}$, for $c \in\left(\frac{2 n}{2(N-1)}, \frac{3 n}{2(N-1)}\right)$ it is $\vec{C} \vec{D}^{*}$, and for $c>\frac{3 n}{2(N-1)}$ it is $\vec{C} \vec{C}$. 
If an H-type introduces $D$, then the following is true.

(i) If he is an interior player, then for $c \lessgtr \frac{n}{2(N-1)}$, the absorbing state is $\vec{D} \vec{D}$, respectively $\vec{C} \vec{C}$.

(ii) If he is a next-to-edge player, then for $c<\frac{n+1}{2(N-1)}$, the absorbing state is $\vec{D} \vec{D}$, for $c \in\left(\frac{n+1}{2(N-1)}, \frac{n+2}{2(N-1)}\right)$, it is $\vec{D} \vec{C}^{*}$, for $c \in\left(\frac{n+2}{2(N-1)}, \frac{2 n-\frac{1}{2}}{2(N-1)}\right)$ it is $\vec{D} \vec{D}$, and for $c>\frac{2 n-\frac{1}{2}}{2(N-1)}$, it is $\vec{C} \vec{C}$.

(iii) If he is an edge player, then for $c<\frac{n+1}{2(N-1)}$, the absorbing state is $\vec{D} \vec{D}$, for $c \in\left(\frac{n+1}{2(N-1)}, \frac{n+2}{2(N-1)}\right)$, it is $\vec{D} \vec{C}^{*}$, for $c \in\left(\frac{n+2}{2(N-1)}, \frac{2 n-\frac{1}{2}}{2(N-1)}\right)$ it is $\vec{D} \vec{D}$, for $c \in$ $\left(\frac{2 n-\frac{1}{2}}{2(N-1)}, \frac{3 n-1}{2(N-1)}\right)$, it is $\vec{C} \vec{D}^{*}$, and for $c>\frac{3 n-1}{2(N-1)}$, it is $\vec{C} \vec{C}$.

Proof: The proof is in the Appendix

The absorbing state $\vec{C} \vec{D}^{*}\left(\vec{D} \vec{C}^{*}\right)$ is identical to $\vec{C} \vec{D}(\vec{D} \vec{C})$ except that edge players of type $L$ play $C$ or cycle between $C$ and $D$ depending on $c$ and $n$. Interestingly, the location of the innovator is irrelevant if the innovator is an $L$ type. In contrast, if the innovator is an $H$-type, the location matters. Finally, the number of agents playing $C$ can decrease in $c$ when the innovation occurs through an $H^{N E_{-}}$or an $H^{E_{\text {-type. }}}$

Figure 1: Maximal segregation, $n=8$.

\begin{tabular}{|c|c|c|c|c|}
\hline$H^{I}$ & $\vec{D} \vec{D}$ & \multicolumn{3}{|c|}{$\vec{C} \vec{C}$} \\
\hline$H^{N E}$ & $\vec{D} \vec{D}$ & $\vec{D} \vec{C}^{*} \quad \vec{D} \vec{D}$ & \multicolumn{2}{|c|}{$\vec{C} \vec{C}$} \\
\hline$H^{E}$ & $\vec{D} \vec{D}$ & $\vec{D} \vec{C}^{*} \quad \vec{D} \vec{D}$ & $\vec{C} \vec{D}^{*}$ & $\vec{C} \vec{C}$ \\
\hline$L$ & \multicolumn{2}{|c|}{$\vec{D} \vec{D}$} & $\vec{C} \vec{C} D^{*}$ & $\vec{C} \vec{C}$ \\
\hline \multicolumn{5}{|l|}{$c$} \\
\hline & $\frac{9}{30} \quad \frac{10}{30}$ & \multicolumn{2}{|c|}{$\frac{23}{30}$} \\
\hline
\end{tabular}

Proposition 5 is illustrated for $n=8$ in Figure 1. It displays the role of the type and the location of the innovator. First, if the cost $c$ is drawn at random, then the population is more likely to end up in the absorbing state $\vec{D} \vec{D}$ when the innovator is an $L$-type than when he is an $H$-type. Second, among the $H$-types the location of the innovator is crucial. If the innovation arises from an $H^{N E_{-}}$or an $H^{E}$-type, then again $\vec{D} \vec{D}$ is more likely than if the innovation arises from an $H^{I}$-type. In this sense, innovations by edge or next-to-edge $H$-types have similar 
consequences for the imitation dynamics as those by $L$-types. Third, the number of agents playing $C$ is non-monotonic in $c$.

Figure 2: Maximal segregation, infinite $n$.

\begin{tabular}{|c|c|c|c|}
\hline$H^{I}$ & $\vec{D} \vec{D}$ & \multicolumn{2}{|l|}{$\vec{C} \vec{C}$} \\
\hline$H^{N E}$ & $\vec{D} \vec{D}$ & \multicolumn{2}{|c|}{$\vec{C} \vec{C}$} \\
\hline$H^{E}$ & $\vec{D} \vec{D}$ & $\vec{C} \vec{D}^{*}$ & $\vec{C} \vec{C}$ \\
\hline$L$ & $\vec{D} \vec{D}$ & $\vec{C} \vec{D}^{*}$ & $\vec{C} \vec{C}$ \\
\hline \multicolumn{4}{|c|}{$c$} \\
\hline & $\frac{1}{4}$ & & \\
\hline
\end{tabular}

An interesting case is the limiting case when there is no finite population effect $(n \rightarrow \infty)$, depicted in Figure 2. In this case the absorbing state $\vec{D} \vec{C}^{*}$ disappears. Without a finite population effect, the difference between an innovation by an $H^{I}$-type and a $H^{N E}$ - or $H^{E}$-type can be seen more easily. For $c \in(1 / 4,1 / 2)$ innovating $H^{E}$ - and $H^{N E}$-types infect $L$-type players, which does not happen if the innovator is an $H^{I}$-type. Note that in this case innovations by $L$-types or $H^{E}$ and $H^{N E}$-types have almost the same consequences for the imitation dynamics.

\subsection{Minimal Segregation of Types}

After having characterized the absorbing states when the population is maximally segregated, we now consider the polar case of a minimally segregated population. This means that we look at a population in which types are located as follows:

\section{HLHLHLHL...HLHLHLHL.}

Evidently, a minimally segregated population consists of double-edge players only. Consequently, there are only two different positions where the strategy $D$ can be introduced, $H^{E E}$ and $L^{E E}$. Again, we assume $n_{H}=n_{L}=n$.

\subsubsection{Absorbing States}

Recall that in a maximally segregated population, all agents of the same type play the same strategy in the absorbing state (with the only exception of edgeplayers of type $L$ in some absorbing states). In contrast, in a minimally segregated 
population, not all agents of the same type will end up playing the same strategy. This requires some additional notation.

We denote by $\tilde{C}_{y_{H}} \tilde{C}_{y_{L}}$ a strategy state where the number of $H$-types and $L$ types playing $C$ is $y_{H}$ and $y_{L}$, respectively. In such a state, all agents of either type who play the same strategy are next to each other. Let us define $\bar{y}$ as the greatest nonnegative odd (even) integer smaller than

$$
2 c(N-1)-n-\frac{1}{2}
$$

if $n$ is even (odd). Note that if $2 c(N-1)-n-\frac{1}{2}<0$, i.e. $c<\frac{n+\frac{1}{2}}{2(N-1)}$, we have $\bar{y}=0$. In this case, the absorbing state is $\tilde{C}_{0} \tilde{C}_{0}$ where all agents play $D$. It is again possible that an absorbing set is attained in which two $L$-types cycle between $D$ and $C$. We denote such an absorbing set by $\tilde{C}_{y_{H}} \tilde{C}_{y_{L}}^{*}$.

Proposition 6 If an $H$-type introduces $D$, then the following is true. If $c<$ $\frac{n+\frac{1}{2}}{2(N-1)}$, the absorbing state is $\tilde{C}_{0} \tilde{C}_{0}$, for $\frac{n+\frac{1}{2}}{2(N-1)}<c<\frac{2 n-\frac{1}{2}}{2(N-1)}$ it is $\tilde{C}_{\bar{y}} \tilde{C}_{\bar{y}-1}^{*}$, for $\frac{2 n-\frac{1}{2}}{2(N-1)}<c<\frac{3 n-1}{2(N-1)}$ it is $\tilde{C}_{n-1} \tilde{C}_{n}$, and for $c>\frac{3 n-1}{2(N-1)}$, it is $\tilde{C}_{n} \tilde{C}_{n}$.

If an L-type introduces $D$, then the following is true. If $c<\frac{n+\frac{1}{2}}{2(N-1)}$, the absorbing state is $\tilde{C}_{0} \tilde{C}_{0}$, for $\frac{n+\frac{1}{2}}{2(N-1)}<c<\frac{2 n+\frac{1}{2}}{2(N-1)}$ it is $\tilde{C}_{\bar{y}} \tilde{C}_{\bar{y}-1}^{*}$, and for $c>\frac{2 n+\frac{1}{2}}{2(N-1)}$ it is $\tilde{C}_{n} \tilde{C}_{n}$.

Proof: The proof is in the Appendix

Several comments are in order here. First, in a minimally segregated population the type of the innovator does not affect the absorbing states significantly. It only matters if $\frac{2 n-\frac{1}{2}}{2(N-1)}<c<\frac{3 n-1}{2(N-1)}$. In this case, the absorbing state is $\tilde{C}_{n-1} \tilde{C}_{n}$ if the innovator is an $H$-type. Second, if the innovator infects its neighbors so that strategy $D$ begins to spread, the spread can be only blocked by $H$-types. Consequently, in any absorbing state where both strategies survive and where more than one player adopts strategy $D$ there will be always one $L$-player more using $D$ than $H$-players, i.e. the absorbing state is of type $\tilde{C}_{\bar{y}} \tilde{C}_{\bar{y}-1}^{*}$. Third, the absorbing state $\tilde{C}_{n-1} \tilde{C}_{n}$ is special because the innovator is a $H$-type, which is not able to infect his $L$-type neighbors. Nevertheless, he continues to use $D$ because strategy $D$ yields a higher payoff in his information set. Consequently, the initial strategy string is stationary.

We illustrate Proposition 6 for $n=8$ in Figure 3 . 
Figure 3: Minimal segregation, $n=8$.

\begin{tabular}{|c|c|c|c|c|}
\hline$H$ & $\tilde{C}_{0} \tilde{C}_{0}$ & $\tilde{C}_{\bar{y}} \tilde{C}_{\bar{y}-1}^{*}$ & $\tilde{C}_{n-1} \tilde{C}_{n}$ & $\tilde{C}_{n} \tilde{C}_{n}$ \\
\hline$L$ & $\tilde{C}_{0} \tilde{C}_{0}$ & $\tilde{C}_{\bar{y}} \tilde{C}_{\bar{y}-1}^{*}$ & \multicolumn{2}{|c|}{$\tilde{C}_{n} \tilde{C}_{n}$} \\
\hline \multicolumn{5}{|l|}{$c$} \\
\hline & & & $\frac{16 \frac{1}{2}}{30}$ & \\
\hline
\end{tabular}

Finally, we also consider the limiting case without finite population effect $(n \rightarrow \infty)$. With $n$ going to infinity, the number $\bar{y}$ goes to infinity, too. So we cannot indicate the respective absorbing state with $\bar{y}$. We use the share of agents playing $C$ in dependence of the costs $c$ instead. Consequently, an absorbing state $\tilde{C}_{4 c-1} \tilde{C}_{4 c-1}$ means that for $c=0.3$ one fifth $(=4 * 0.3-1)$ of the $H$-types and one fifth of the $L$-types play $C$.

Figure 4: Minimal segregation, infinite $n$.

\begin{tabular}{|c|c|c|c|}
\hline$H$ & $\tilde{C}_{0} \tilde{C}_{0}$ & $\tilde{C}_{4 c-1} \tilde{C}_{4 c-1}^{*}$ & $\tilde{C}_{1} \tilde{C}_{1}$ \\
\hline$L$ & $\tilde{C}_{0} \tilde{C}_{0}$ & $\tilde{C}_{4 c-1} \tilde{C}_{4 c-1}^{*}$ & $\tilde{C}_{1} \tilde{C}_{1}$ \\
\hline$c$ & & & + \\
\hline & & & $\frac{3}{4}$ \\
\hline
\end{tabular}

From Figure 4 we can see that without finite population effect the type of innovator is irrelevant. If $c<1 / 4$, then all players will end up using $D$. For $1 / 4<c<1 / 2$, the fraction of $H$-types and $L$-types using $C$ is equal and strictly increasing in $c$. Finally, for $c>1 / 2$, all agents play $C$. This is interesting because with global information in the limiting case when the number of players is large we get the result that if $c \lessgtr \frac{1}{2}$, the absorbing state is $\vec{D} \vec{D}(\vec{C} \vec{C})$ (see Proposition 3 ). Thus, local information reduces the spread of the strategy $D$ if agents are minimally segregated.

\subsection{Maximal versus Minimal Segregation}

We conclude this section with a comparison of the absorbing states of a maximally and of a minimally segregated population. For this comparison we calculate the 
expected share of agents playing $C$ in the absorbing state when each agent is equally likely to innovate $D$. We focus on large populations $(n \rightarrow \infty)$ such that finite population effects can be neglected. Another consequence of this assumption is that the role of edge players (of which there are but two of each type in the maximally segregated population and none in the minimally segregated population) becomes negligible.

Figure 5 depicts the expected shares of agents playing $C$ in the absorbing states for maximally and minimally segregated populations. The left-hand panel displays the shares for both populations when $D$ is introduced by an $H$-type, while the right-hand panel displays these shares if innovation occurs by an $L$ type.

Figure 5: Comparing maximal and minimal segregation, infinite $n$.
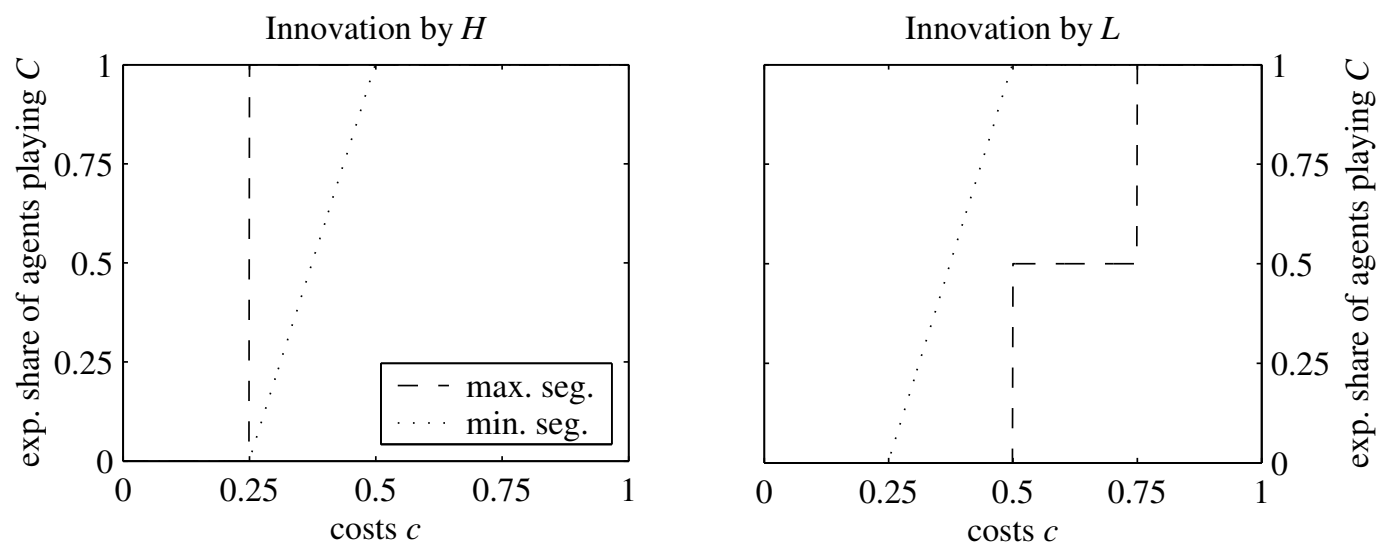

There are two things worth pointing out. First, in a minimally segregated population the location of the innovator does not matter. Consequently, for a minimally segregated population the graph depicting the share of agents playing $C$ (the dotted curves in Figure 5) is identical in both panels. Second, if the innovator is a low type, then a minimally segregated population is more resistant against the cheating strategy $D$ than a maximally segregated population. The reason for this result is that in a minimally segregated population each low type (including the innovator) is surrounded by two high types who are less prone to imitate $D$. In contrast, if the innovator is a high type, then a maximally segregated population is more resistant against $D$ than a minimally segregated 
population. The reason for this result is that in a maximally segregated population the innovator is again surrounded by high types which are less prone to imitate $D$.

Proposition 7 Consider a large population $(n \rightarrow \infty)$. Then, if each agent is equally likely to innovate the cheating strategy $D$, the following is true.

If $c<\frac{2}{8}$, all agents play $D$ in the absorbing state for both distributions of types. If $\frac{2}{8}<c<\frac{3}{8}$, more agents play $D$ in a minimally segregated population than in a maximally segregated one.

If $\frac{3}{8}<c<\frac{6}{8}$, less agents play $D$ in a minimally segregated population than in a maximally segregated one.

Finally, if $c>\frac{6}{8}$, all agents play $C$ in the absorbing state for both distributions of types.

These results follow from Propositions 5 and 6. They are depicted in Figure 6.

Figure 6: Comparing maximal and minimal segregation, infinite $n$.

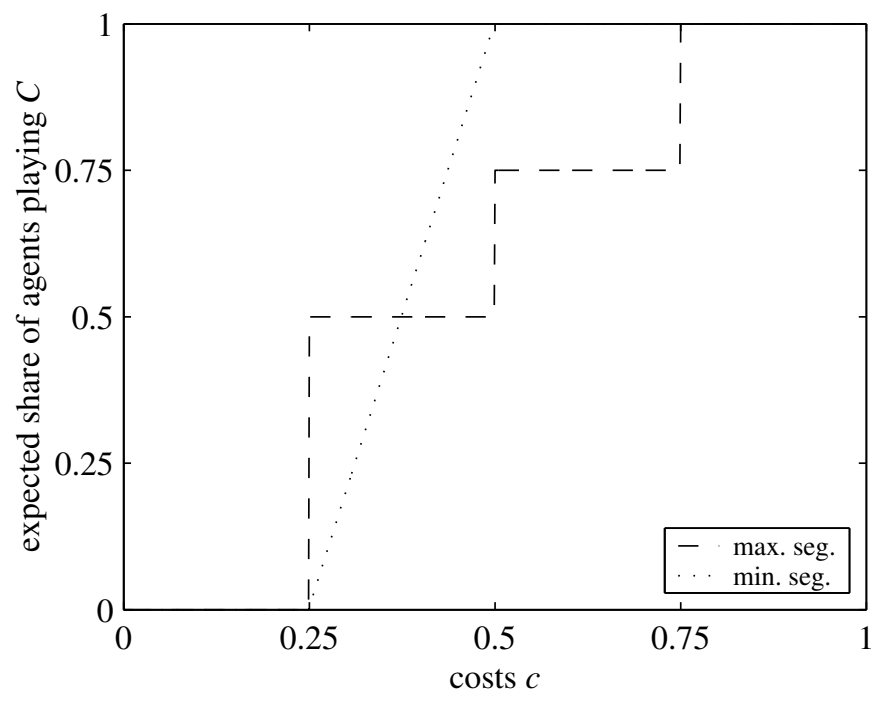

For $c \in\left(\frac{3}{8}, \frac{6}{8}\right)$ the minimally segregated population exhibits a higher share of agents playing $C$ in the absorbing state than the maximally segregated population. The reason for this is that being located between $L$-types, the $H$-types are able to block the spread of strategy $D$. However, for sufficiently low cheating 
costs, i.e. for $c \in\left(\frac{2}{8}, \frac{3}{8}\right)$, the maximally segregated population exhibits a higher share of agents playing $C$. The reason is that in a maximally segregated population $L$-types never observe $D$ if the innovator is a $H$-type. In contrast, in a minimally segregated population, if $D$ is introduced by a $H$-type, two $L$-type will observe it. And since $L$-types are more likely to be infected than $H$-types the population share of agents playing $D$ is larger with minimal segregation.

\section{Mutations}

In this section we introduce mutations. In each period, after imitations have occurred, each agent's strategy changes with a small probability $\varepsilon .{ }^{7}$ As mentioned before, the imitation dynamics is a Markov chain evolving over the strategy space $S$. A probability distribution over $S$ in time $t$ is represented as a row vector $\nu$ which is an element of the $2^{N}$-dimensional simplex. The simplex $\Sigma_{N}$ is the set

$$
\Sigma_{N}=\left\{\nu \in \mathbb{R}^{2^{N}} \mid \nu_{i} \geq 0 \text { and } \sum_{i} \nu_{i}=1 \text { for } i=1,2, \ldots, 2^{N}\right\} .
$$

The process evolves according to $\nu_{t+1}=\nu_{t} P$, where $P$ is the transition probability matrix defined in Section 2.3. Now that agents' strategies change with probability $\varepsilon$ after imitation, the transition probability $p_{i j}$ is positive for all $i$ and $j$, i.e. the Markov chain is regular. Thus, there exists a unique probability distribution $\mu \in \Sigma_{N}$ such that ${ }^{8}$

$$
\mu P=\mu
$$

The vector $\mu$ is the unique stationary distribution of the regular Markov process, which does not depend on the initial probability distribution. The stationary distribution $\mu$ is stable, i.e.

$$
\lim _{t \rightarrow \infty} \nu P^{t}=\mu \quad \forall \nu \in \Sigma_{N}
$$

¿From the law of large numbers for regular Markov chains we have

$$
E\left[\frac{1}{T} \sum_{t=1}^{T} z_{i, t}\right] \rightarrow \mu_{i} \text { with } z_{i, t}=\left\{\begin{array}{l}
1 \text { if } s_{t}=i \\
0 \text { otherwise }
\end{array}\right.
$$

\footnotetext{
${ }^{7}$ See Kandori et al. (1993, p.38) or Ellison (1993, p.1050) for an interpretation of $\varepsilon$.

${ }^{8}$ See e.g. Kemeny and Snell (1960), Theorem 4.1.6.(b).
} 
as $T$ tends to infinity. ${ }^{9}$ Therefore, the probabilities in the limiting distribution can be interpreted as average share of time the process spends in a given state. The transition matrix $P(\varepsilon)$ and the stationary distribution $\mu(\varepsilon)$ depend on $\varepsilon$. The stationary and stable probability distribution $\mu(\varepsilon)$ describes the long-run behavior of the imitation dynamics with mutations. Since we are interested in the imitation dynamics for small $\varepsilon$, we consider the limiting distribution $\mu^{*}$ :

$$
\mu^{*}=\lim _{\varepsilon \rightarrow 0} \mu(\varepsilon) .
$$

The limiting distribution $\mu^{*}$, if it exists, depends on the parameter values $\{c, n\}$ of our model. Even for very small populations, evaluating $\mu^{*}$ involves solving a large equation system of $2^{2 n}$ variables. Instead of finding $\mu(\varepsilon)$ explicitly and taking the limit for $\varepsilon \rightarrow 0$, we approximate $\mu^{*}$ numerically. We will describe $\mu^{*}$ for a maximally segregated population and the smallest population size $(n=5)$ that provides all relevant positions of innovation (i.e. $H^{I}, H^{N E}, H^{E}$, and $L$ ) as described previously. Our simulations suggest that the following results hold. ${ }^{10}$

Conjecture 1 If a population is maximally segregated and the individual mutation rate $\varepsilon$ goes to zero, then our simulations indicate the following. If $c<\frac{n}{2(N-1)}$, we have $\operatorname{Pr}(\vec{D} \vec{D})=1$, for $\frac{n}{2(N-1)}<c<\frac{2 n-2}{2(N-1)}$ then $\operatorname{Pr}\left(\vec{D} \vec{C}^{*}\right)=1$, for $\frac{2 n-2}{2(N-1)}<$ $c<\frac{2 n+2}{2(N-1)}$, then $\operatorname{Pr}(M)=1$ where $M=\left\{\vec{D} \vec{D}, \vec{D} \vec{C}^{*}, \vec{D} \vec{C}, \vec{C} \vec{D}, \vec{C} \vec{D}^{*}, \vec{C} \vec{C}\right\}$, for $\frac{2 n+2}{2(N-1)}<c<\frac{3 n}{2(N-1)}$ then $\operatorname{Pr}\left(\vec{C} \vec{D}^{*}\right)=1$, and for $c>\frac{3 n}{2(N-1)}$, then $\operatorname{Pr}(\vec{C} \vec{C})=1$.

We illustrate our conjecture for $n=5$ and $n \rightarrow \infty$ in the following Figures.

Figure 7: Maximal segregation with mutations, $n=5$.

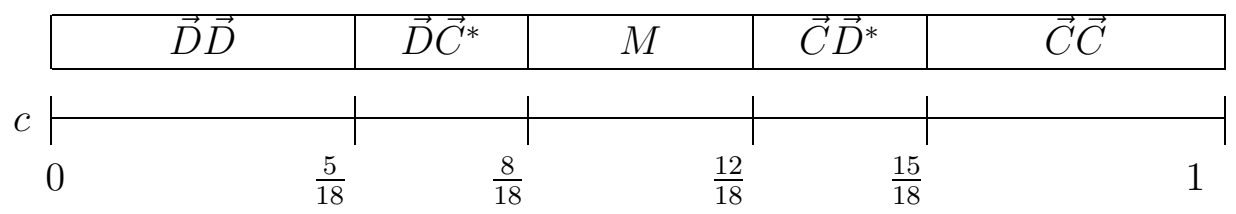

Figure 7 demonstrates that the absorbing state $\vec{D} \vec{D}$ arising from $c \in\left(\frac{n+2}{2(N-1)}, \frac{2 n-\frac{1}{2}}{2(N-1)}\right)$ in the model without mutations disappears. Nevertheless,

\footnotetext{
${ }^{9}$ See e.g. Kemeny and Snell (1960), Theorems 4.1.6.(a) and 4.2.1.

${ }^{10}$ The Matlab file with our simulations can be downloaded at http://www.unibas.ch/wwz/witheo/aleks/doping/Doping.htm.
} 
we still observe non-monotonicity because $\vec{D} \vec{D}$ is an element of the absorbing set $M$ and is played with strictly positive probability.

Again, an interesting case is the limiting case when $n \rightarrow \infty$, depicted in Figure 8.

Figure 8: Maximal segregation with mutations, infinite $n$.

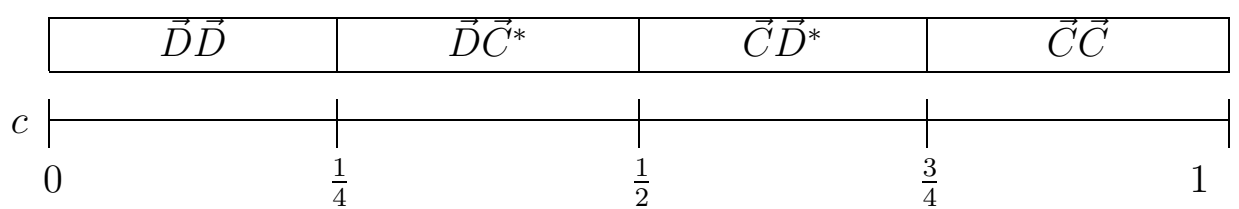

For $c<\frac{1}{4}$, the model with mutations and the deterministic model both give way to the absorbing state $\vec{D} \vec{D}$. If $\frac{1}{4}<c<\frac{1}{2}$, the absorbing state $\vec{D} \vec{C}^{*}$ is reached with probability 1 in the model with mutations. In the deterministic model, on the other hand, the absorbing state is $\vec{C} \vec{C}$ if the innovator is an $H^{I}$-type and $\vec{D} \vec{D}$ otherwise. From this we conclude that our results in the deterministic model are not robust with respect to mutations. This is not so surprising, since due to the imitation rule, agents can only imitate strategies they observe in their information set. Thus, in the absorbing states $\vec{C} \vec{C}$ and $\vec{D} \vec{D}$, the population is locked in. This cannot occur with mutations. For $\frac{1}{2}<c<\frac{3}{4}$, the model without mutations exhibits another lock-in, which arises if the innovation occurs through an $H^{I}$ - or an $H^{N E}$-type. For $c>\frac{3}{4}$, the model with mutations and the deterministic model have the same absorbing state, $\vec{C} \vec{C}$. 


\section{Conclusions}

In this paper we consider an evolutionary game where agents can either play fair or cheat. A cheating agent bears some direct utility cost but wins the stage game against a fair playing agent with certainty. The paper extends the standard framework of an evolutionary game by introducing heterogeneity among agents. There are two types of agents where high types have a natural advantage over low types, i.e. they win the stage game with certainty if both agents apply the same strategy. A consequence of the two type assumption is that there are two stage games. If two agents of equal type are matched, the stage game is a standard Prisoner's Dilemma. If they are of opposite type, then the stage game is an asymmetric game similar to a matching penny game.

We consider the imitation dynamics when initially all agents play fair and one agent invents the cheating strategy. In contrast to the standard framework, with heterogenous agents the absorbing state depends on the location and the type of the innovator, the distribution of types on the circle, and the information (i.e. local or global) available to the agents. The paper shows how these factors determine whether the innovator is able to infect his neighbors and eventually to contaminate the entire population.

We focus on two distribution of types: maximal (equal types are located next to each other) and minimal (agents have always neighbors of opposite type) segregation. The following results emerge from the model. First, the population is more likely to end up in an absorbing state where all agents cheat if the innovator is a low type. Second, if the innovator is a low type, then a minimally segregated population is more resistant against the cheating strategy than a maximally segregated population. Third, in contrast, if the innovator is a high type, a maximally segregated population is more resistant against the cheating strategy than a minimally segregated population. 


\section{Appendix}

\section{Proof of Proposition 3}

If the innovating agent is an $H$-type, the initial numbers of clean agents are $y_{H}=n_{H}-1$ and $y_{L}=n_{L}$. If the innovator is an $L$-type, these numbers are $y_{H}=n_{H}$ and $y_{L}=n_{L}-1$. We first notice that due to global information we have that

$$
\Delta_{H}\left(y_{H}, y_{L}\right)=\Delta_{L}\left(y_{H}, y_{L}\right) \text {. }
$$

We therefore drop the type group index of the decision terms.

If the innovation arises from an $H$-type, the decision term for all agents is

$$
\begin{aligned}
\Delta\left(n_{H}-1, n_{L}\right)= & u_{H}\left(1, n_{H}-1, n_{L}\right) \\
& -\frac{\left(n_{H}-1\right) u_{H}\left(0, n_{H}-1, n_{L}\right)+n_{L} u_{L}\left(0, n_{H}-1, n_{L}\right)}{N-1} .
\end{aligned}
$$

From (4) and (5) we get

$$
\begin{aligned}
& u_{H}\left(d_{i}, n_{H}-1, n_{L}\right)=\frac{N+n_{L}-2}{2(N-1)}+d_{i}\left(\frac{n_{H}}{2(N-1)}-c\right) \text { and } \\
& u_{L}\left(d_{i}, n_{H}-1, n_{L}\right)=\frac{n_{L}-1}{2(N-1)}+d_{i}\left(\frac{N+n_{H}-2}{2(N-1)}-c\right), \text { respectively. }
\end{aligned}
$$

Using this information we get

$$
\Delta\left(n_{H}-1, n_{L}\right)=\frac{n_{L}+n_{H}}{2\left(n_{L}+n_{H}-1\right)}-c .
$$

If the innovation arises from an $L$-type agent, the difference in the average payoffs is

$$
\begin{aligned}
\Delta\left(n_{H}, n_{L}-1\right)= & u_{L}\left(1, n_{H}, n_{L}-1\right) \\
& -\frac{n_{H} u_{H}\left(0, n_{H}, n_{L}-1\right)+\left(n_{L}-1\right) u_{L}\left(0, n_{H}, n_{L}-1\right)}{N-1} .
\end{aligned}
$$

From (4) and (5) we get

$$
\begin{aligned}
u_{H}\left(d_{i}, n_{H}, n_{L}-1\right) & =\frac{N+n_{L}-3}{2(N-1)}+d_{i}\left(\frac{n_{H}+2}{2(N-1)}-c\right) \text { and } \\
u_{L}\left(d_{i}, y_{H}, n_{L}-1\right) & =\frac{n_{L}-2}{2(N-1)}+d_{i}\left(\frac{N+n_{H}}{2(N-1)}-c\right), \text { respectively. }
\end{aligned}
$$


Using this information we get

$$
\Delta\left(n_{H}, n_{L}-1\right)=\frac{n_{L}+n_{H}}{2\left(n_{L}+n_{H}-1\right)}-c .
$$

Note that

$$
\Delta\left(n_{H}, n_{L}-1\right)=\Delta\left(n_{H}-1, n_{L}\right) .
$$

Consequently, the origin of the innovation does not matter if agents have no type information.

\section{Proof of Proposition 4}

If agents have complete information, they have both strategies available at any point in time, distinguish types and are able to calculate the payoff they would have gotten having played the other strategy. Under these circumstances the imitation rule (2) can be interpreted as a best response dynamics. As before, we can define suitable decision terms. We have

$$
\begin{aligned}
& \Delta_{H}\left(y_{H}, y_{L}\right)=u_{H}\left(1, y_{H}, y_{L}\right)-u_{H}\left(0, y_{H}, y_{L}\right)=\frac{n_{H}+2 n_{L}-2 y_{L}}{2(N-1)}-c, \\
& \Delta_{L}\left(y_{H}, y_{L}\right)=u_{L}\left(1, y_{H}, y_{L}\right)-u_{L}\left(0, y_{H}, y_{L}\right)=\frac{n_{L}+2 y_{H}}{2(N-1)}-c
\end{aligned}
$$

At the end of the first period the agents decide either to play

- $\vec{D} \vec{D}$, if $c<\frac{n_{H}}{2(N-1)}$ when $H$ innovates (from $\Delta_{H}\left(n_{H}-1, n_{L}\right)>0$ ) or if $c<\frac{n_{H}+2}{2(N-1)}$ when $L$ innovates (from $\Delta_{H}\left(n_{H}, n_{L}-1\right)>0$ )

- $\vec{C} \vec{D}$, if $\frac{n_{H}}{2(N-1)}<c<\frac{2 n_{H}+n_{L}-2}{2(N-1)}$ when $H$ innovates (from $\Delta_{H}\left(n_{H}-1, n_{L}\right)<0$ and $\left.\Delta_{L}\left(n_{H}-1, n_{L}\right)>0\right)$

or if $\frac{n_{H}+2}{2(N-1)}<c<\frac{2 n_{H}+n_{L}}{2(N-1)}$ when $L$ innovates (from $\Delta_{H}\left(n_{H}, n_{L}-1\right)<0$ and $\left.\Delta_{L}\left(n_{H}, n_{L}-1\right)>0\right)$

- $\vec{C} \vec{C}$ for $\frac{2 n_{H}+n_{L}-2}{2(N-1)}<c$ when $H$ innovates (from $\Delta_{L}\left(n_{H}-1, n_{L}\right)<0$ ) or if $\frac{2 n_{H}+n_{L}}{2(N-1)}<c$ when $L$ innovates (from $\Delta_{L}\left(n_{H}, n_{L}-1\right)<0$ ).

Note that $\vec{D} \vec{C}$ will not be played in the second period because of $\Delta_{H}\left(n_{H}-\right.$ $\left.1, n_{L}\right)<\Delta_{L}\left(n_{H}-1, n_{L}\right)$ and $\Delta_{H}\left(n_{H}, n_{L}-1\right)<\Delta_{L}\left(n_{H}, n_{L}-1\right)$. What kind of $\Delta_{H}$ and $\Delta_{L}$ do these strategy states imply? 
- All play $C$ (situation $\vec{C} \vec{C}$ ), implies

$$
\Delta_{H}\left(n_{H}, n_{L}\right)=\frac{n_{H}}{2(N-1)}-c \quad \text { and } \quad \Delta_{L}\left(n_{H}, n_{L}\right)=\frac{2 n_{H}+n_{L}}{2(N-1)}-c,
$$

- all play $D$ (situation $\vec{D} \vec{D})$, implies

$$
\Delta_{H}(0,0)=\frac{n_{H}+2 n_{L}}{2(N-1)}-c \quad \text { and } \quad \Delta_{L}(0,0)=\frac{n_{L}}{2(N-1)}-c,
$$

- group $H$ plays $C$ and group $L$ plays $D$ (situation $\vec{C} \vec{D}$ ), implies

$$
\Delta_{H}\left(n_{H}, 0\right)=\frac{n_{H}+2 n_{L}}{2(N-1)}-c \quad \text { and } \quad \Delta_{L}\left(n_{H}, 0\right)=\frac{2 n_{H}+n_{L}}{2(N-1)}-c
$$

- group $H$ plays $D$ and group $L$ plays $C$ (situation $\vec{D} \vec{C}$ ), implies

$$
\Delta_{H}\left(0, n_{L}\right)=\frac{n_{H}}{2(N-1)}-c \quad \text { and } \quad \Delta_{L}\left(0, n_{L}\right)=\frac{n_{L}}{2(N-1)}-c .
$$

From (13) and (14) it is clear that $\vec{C} \vec{C}$ is an equilibrium strategy state if

$$
c>\max \left\{\frac{n_{H}+2 n_{L}}{2(N-1)}, \frac{2 n_{H}+n_{L}}{2(N-1)}\right\}
$$

and $\vec{D} \vec{D}$ is an absorbing state if

$$
c<\min \left\{\frac{n_{H}}{2(N-1)}, \frac{n_{L}}{2(N-1)}\right\} .
$$

If $c$ does not satisfy one of these two conditions we can work out the following cycle by using the $\Delta$-functions above:

$$
\ldots \rightarrow \vec{C} \vec{D} \rightarrow \vec{D} \vec{D} \rightarrow \vec{D} \vec{C} \rightarrow \vec{C} \vec{C} \rightarrow \vec{C} \vec{D} \rightarrow \vec{D} \vec{D} \rightarrow \vec{D} \vec{C} \rightarrow \ldots
$$

For $c$ in an interval $\left[\min \left\{\frac{n_{H}}{2(N-1)}, \frac{n_{L}}{2(N-1)}\right\}, \max \left\{\frac{n_{H}+2 n_{L}}{2(N-1)}, \frac{2 n_{H}+n_{L}}{2(N-1)}\right\}\right]$ we will observe an absorbing set of strategy states. The imitation dynamics cycles between the four strategy states $\{\vec{C} \vec{D}, \vec{D} \vec{D}, \vec{D} \vec{C}, \vec{C} \vec{C}\}$, where a single agent plays $C$ for two periods followed by $D$ for two periods etc. The strategy states specific to the two type groups are shifted in time by one or three periods, depending on where in the cycle we start to count. 


\section{Decision Terms for Edge Players}

The decision terms for an edge player of type $H$ are

$$
\begin{aligned}
\Delta_{H^{E}}^{1}\left(y_{H}, y_{L}\right) & =u_{H}\left(1, y_{H}, y_{L}\right)-u_{L}\left(0, y_{H}, y_{L}\right) \\
& =\frac{n_{H}+2 n_{L}+y_{H}-y_{L}}{2(N-1)}-c \\
\Delta_{H^{E}}^{2}\left(y_{H}, y_{L}\right) & =u_{H}\left(1, y_{H}, y_{L}\right)-\frac{1}{2}\left[u_{H}\left(0, y_{H}, y_{L}\right)+u_{L}\left(0, y_{H}, y_{L}\right)\right] \\
& =\frac{n_{H}+2 n_{L}+\frac{1}{2} y_{H}-\frac{3}{2} y_{L}}{2(N-1)}-c, \\
\Delta_{H^{E}}^{3}\left(y_{H}, y_{L}\right) & =\frac{1}{2}\left[u_{H}\left(1, y_{H}, y_{L}\right)+u_{L}\left(1, y_{H}, y_{L}\right)\right]-u_{H}\left(0, y_{H}, y_{L}\right) \\
& =\frac{\frac{1}{2} n_{H}+\frac{3}{2} n_{L}+\frac{1}{2} y_{H}-\frac{3}{2} y_{L}}{2(N-1)}-c, \\
\Delta_{H E}^{4}\left(y_{H}, y_{L}\right) & =u_{L}\left(1, y_{H}, y_{L}\right)-u_{H}\left(0, y_{H}, y_{L}\right) \\
& =\frac{n_{L}+y_{H}-y_{L}}{2(N-1)}-c .
\end{aligned}
$$

For an edge player of type $L$ we get

$$
\begin{aligned}
\Delta_{L^{E}}^{1}\left(y_{H}, y_{L}\right) & =u_{L}\left(1, y_{H}, y_{L}\right)-u_{H}\left(0, y_{H}, y_{L}\right) \\
& =\frac{n_{L}+y_{H}-y_{L}}{2(N-1)}-c \\
\Delta_{L^{E}}^{2}\left(y_{H}, y_{L}\right) & =u_{L}\left(1, y_{H}, y_{L}\right)-\frac{1}{2}\left[u_{H}\left(0, y_{H}, y_{L}\right)+u_{L}\left(0, y_{H}, y_{L}\right)\right] \\
& =\frac{n_{L}+\frac{3}{2} y_{H}-\frac{1}{2} y_{L}}{2(N-1)}-c, \\
\Delta_{L^{E}}^{3}\left(y_{H}, y_{L}\right) & =\frac{1}{2}\left[u_{L}\left(1, y_{H}, y_{L}\right)+u_{H}\left(1, y_{H}, y_{L}\right)\right]-u_{L}\left(0, y_{H}, y_{L}\right) \\
& =\frac{\frac{1}{2} n_{H}+\frac{3}{2} n_{L}+\frac{3}{2} y_{H}-\frac{1}{2} y_{L}}{2(N-1)}-c, \\
\Delta_{L^{E}}^{4}\left(y_{H}, y_{L}\right) & =u_{H}\left(1, y_{H}, y_{L}\right)-u_{L}\left(0, y_{H}, y_{L}\right) \\
& =\frac{n_{H}+2 n_{L}+y_{H}-y_{L}}{2(N-1)}-c .
\end{aligned}
$$




\section{Decision Terms for Double-Edge Players}

For a double-edge player of type $H$ the decision terms are

$$
\begin{aligned}
\Delta_{H^{E E}}^{1}\left(y_{H}, y_{L}\right) & =\frac{n_{L}+y_{H}-y_{L}}{2(N-1)}-c, \\
\Delta_{H^{E E}}^{2}\left(y_{H}, y_{L}\right) & =\frac{n_{L}+\frac{3}{2} y_{H}-\frac{1}{2} y_{L}}{2(N-1)}-c, \\
\Delta_{H^{E E}}^{3}\left(y_{H}, y_{L}\right) & =\frac{\frac{1}{2} n_{H}+\frac{3}{2} n_{L}+\frac{3}{2} y_{H}-\frac{1}{2} y_{L}}{2(N-1)}-c, \\
\Delta_{H^{E E}}^{4}\left(y_{H}, y_{L}\right) & =\frac{n_{H}+2 n_{L}+y_{H}-y_{L}}{2(N-1)}-c,
\end{aligned}
$$

and for a double-edge player of type $L$ they are

$$
\begin{aligned}
\Delta_{L^{E E}}^{1}\left(y_{H}, y_{L}\right) & =\frac{n_{H}+2 n_{L}+y_{H}-y_{L}}{2(N-1)}-c, \\
\Delta_{L^{E E}}^{2}\left(y_{H}, y_{L}\right) & =\frac{n_{H}+2 n_{L}+\frac{1}{2} y_{H}-\frac{3}{2} y_{L}}{2(N-1)}-c, \\
\Delta_{L^{E E}}^{3}\left(y_{H}, y_{L}\right) & =\frac{\frac{1}{2} n_{H}+\frac{3}{2} n_{L}+\frac{1}{2} y_{H}-\frac{3}{2} y_{L}}{2(N-1)}-c, \\
\Delta_{L^{E E}}^{4}\left(y_{H}, y_{L}\right) & =\frac{n_{L}+y_{H}-y_{L}}{2(N-1)}-c .
\end{aligned}
$$

\section{Proof of Proposition 5}

We accomplish the proof of Proposition 5 in two parts. We first consider the absorbing states when an $L$-type introduces $D$.

\section{L-type introduces D.}

In the following we assume that the innovator is an interior player $L^{I}$. The absorbing states are the same when an $L^{N E}$ or an $L^{E}$ innovates $D$.

(a) If $c>\frac{3 n}{2(N-1)}$, in the absorbing state all players play $C$ because from (5) $\Delta_{L^{I}}(n, n-1)<0$. Consequently, $D$ dies out immediately.

(b) If $c \in\left(\frac{2 n}{2(N-1)}, \frac{3 n}{2(N-1)}\right)$ the absorbing state is $\vec{C} \vec{D}^{*}$. This interval can be divided into two subintervals. First, if $c \in\left(\frac{\frac{5}{2} n-1}{2(N-1)}, \frac{3 n}{2(N-1)}\right)$ in the absorbing state all $L^{I}$ play $D$, and the two $L^{E}$ and all $H$-types play $C$. If $c<\frac{3 n}{2(N-1)}$, interior players of type $L$ imitate $D$ because $\Delta_{L^{I}}\left(n, y_{L}\right)=\frac{3 n}{2(N-1)}-c>0$. (Note that $\Delta_{L^{I}}\left(n, y_{L}\right)$ does not depend on $\left.y_{L}\right)$. At one point in time, an $L^{E}$ observes $D$. He adopts it if $\Delta_{L^{E}}^{2}\left(n, y_{L}\right)=\frac{\frac{5}{2} n-\frac{1}{2} y_{L}}{2(N-1)}-c>0$. Since $\Delta_{L^{E}}^{2}$ depends negatively on $y_{L}$, 
$\Delta_{L^{E}}^{2}$ is increasing when the number of $L^{I}$ using $D$ increases. Thus, $\Delta_{L^{E}}^{2}\left(n, y_{L}\right)$ is maximal when $y_{L}=2$. Consequently, if $\frac{\frac{5}{2} n-1}{2(N-1)}-c>0$, the edge players of type $L$ never adopt $D$, and an absorbing state is reached.

Second, if $c \in\left(\frac{2 n}{2(N-1)}, \frac{\frac{5}{2} n-1}{2(N-1)}\right)$, in the absorbing state all $H$ play $C$, all $L^{I}$ play $C$, and the $L^{E}$ cycle between $D$ and $C$. If $c<\frac{\frac{5}{2} n-1}{2(N-1)}$, an $L^{E}$-type imitates $D$. The first $H^{E}$-agent observing $D$ has the decision term $\Delta_{H^{E}}^{4}\left(y_{H}, y_{L}\right)=\frac{n+y_{H}-y_{L}}{2(N-1)}-c$. He adopts $D$ if $\Delta_{H^{E}}^{4}\left(y_{H}, y_{L}\right)>0$. Since all $H$-types still use $C$ we have $y_{H}=n$. Let us first assume that $c$ is such that he does not imitate $D$. Then, the number $L$-types adopting $D$ is increasing because their decision term does not depend on $y_{L}$. Consequently, $y_{L}$ decreases to 0 implying $\Delta_{H^{E}}^{4}(n, 0)=\frac{N}{2(N-1)}-c .{ }^{11}$ Thus, if $\frac{2 n}{2(N-1)}<c<\frac{\frac{5}{2} n-1}{2(N-1)}$ all $H$-types play $C$ and the $L^{E}$-types cycle between $C$ and $D$ because $\Delta_{L^{E}}^{1}(n, 0)<0$ and $\Delta_{L^{E}}^{2}(n, 2)>0$.

(c) If $c<\frac{2 n}{2(N-1)}$, in the absorbing state all players play $D$. If $c<\frac{2 n}{2(N-1)}$, $\Delta_{H^{E}}^{4}\left(y_{H}, y_{L}\right)>0$. Then, from (9) all other $H$-types will also imitate $D$. Note that $L$-types continue to use $D$ because they do not observe strategy $C$ in their information set anymore. Consequently, in the absorbing state all players play $D$.

\section{H-type introduces D.}

If an $H^{I}$ introduces $D$, we have to consider the intervals $c \in\left(0, \frac{n}{2(N-1)}\right)$ and $c \in\left(\frac{n}{2(N-1)}, 1\right)$.

(a) If $c>\frac{n}{2(N-1)}$, then $\Delta_{H^{I}}(n-1, n)<0$ and $D$ is extinguished in the second period. The absorbing state is $\vec{C} \vec{C}$.

(b) If $c<\frac{n}{2(N-1)}$, then $\Delta_{H^{I}}(n-1, n)>0$, and the $H^{I}$ that observe $D$ will play it in the second period. In the following periods, more and more $H^{I}$ will switch to $D$ since $\Delta_{H^{I}}$ is unchanged as long as only $H$-types observe $D$. At some point an $H^{E}$ will observe $D$. His decision term is $\Delta_{H^{E}}^{2}$, which from (9), is always higher than $\Delta_{H^{I}}(n-1, n)$. So he will adopt. The next player to

\footnotetext{
${ }^{11}$ Note that $y_{L}$ can only become 1 and not 0 if $n$ is even. Start counting outward from an $L^{I}$ inventing $D$. Since $n$ is even, it takes an uneven number of periods from the period the the first $L^{E}$ observes $D$ until the second $L^{E}$ observes $D$. As explained in the text, the $L^{E}$ cycle between $C$ and $D$ for the interval under consideration. Hence the two $L^{E}$ do not play the same strategies in any given period. This shifts the lower bound of the respective interval to $c \in\left(\frac{2 n-1}{2(N-1)}, \frac{\frac{5}{2} n-1}{2(N-1)}\right)$ for even $n$. In the Proposition we state the result for odd $n$.
} 
consider is $L^{E}$. His decision term is $\Delta_{L^{E}}^{4}\left(y_{H}, n\right)$. He also adopts $D$ because $\Delta_{L^{E}}^{4}\left(y_{H}, y_{L}\right)>\Delta_{H^{I}}\left(y_{H}, y_{L}\right)>0$. Now all $L^{I}$ adopt $D$ too, since $\Delta_{L^{I}}>0$ for $c<\frac{n}{n(N-1)}$. The last question we have to answer is whether the decreasing $y_{H}$ or $y_{L}$ can stop the spreading in either of the two groups. For the $H$-types this is not the case because $\Delta_{H^{I}}$ is decreasing in $y_{L}$. For the $L$-types we find that if $c<\frac{n}{2(N-1)}$, then $\Delta_{L^{I}}$ is always positive. Consequently, if $c<\frac{n}{2(N-1)}$, the absorbing state is $\vec{D} \vec{D}$.

(ii)

If an $H^{N E}$ invents $D$, one $H^{I}$ and one $H^{E}$ observe $D$. For this reason, it is convenient to divide the proof into three subsections: we consider the cost intervals $\left(0, \frac{n}{2(N-1)}\right),\left(\frac{n}{2(N-1)}, \frac{2 n-\frac{1}{2}}{2(N-1)}\right)$, and $\left(\frac{2 n-\frac{1}{2}}{2(N-1)}, 1\right)$ separately.

(a) If $c<\frac{n}{2(N-1)}$, one can show that all decision terms are strictly positive. Consequently, the absorbing state is $\vec{D} \vec{D}$.

(b) For $\frac{n}{2(N-1)}<c<\frac{2 n-\frac{1}{2}}{2(N-1)}$, we find several absorbing states. We derive them one by one. In the first period we have $\Delta_{H^{E}}^{2}(n-1, n)>0$ but $\Delta_{H^{I}}(n-1, n)<0$. In period $t=2, H^{E}$ is thus the only agent playing $D$, since $H^{N E}$ who invented $D$ abandons it (he decides according to $\Delta_{H^{I}}$ ) and $H^{I}$ does not adopt it. So now $L^{E}, H^{E}$ and $H^{N E}$ face the decision to imitate $D$ or not. Their decision terms are $\Delta_{L^{E}}^{4}, \Delta_{H^{E}}^{2}$ and $\Delta_{H^{I}}$, valued at $(n-1, n)$. With $c \in\left(\frac{n}{2(N-1)}, \frac{2 n-\frac{1}{2}}{2(N-1)}\right)$ we have

$$
\begin{aligned}
\Delta_{L^{E}}^{4}(n-1, n) & =\frac{3 n-1}{2(N-1)}-c>0 \\
\Delta_{H^{E}}^{2}(n-1, n) & =\frac{2 n-\frac{1}{2}}{2(N-1)}-c>0 \\
\Delta_{H^{I}}(n-1, n) & =\frac{n}{2(N-1)}-c<0 .
\end{aligned}
$$

So we know that in period $t=3 L^{E}$ as well as $H^{E}$ play $D$ and $L^{I}, L^{E}, H^{E}$, and $H^{I}$ have to decide to adopt or abandon $D$. By looking at these four decision terms $\left(\Delta_{L^{I}}, \Delta_{L^{E}}^{3}, \Delta_{H^{E}}^{3}\right.$, and $\left.\Delta_{H^{I}}\right)$ evaluated at $(n-1, n-1)$, it becomes clear 
that the process now is not unique for the whole interval of $c \in\left(\frac{n}{2(N-1)}, \frac{2 n-\frac{1}{2}}{2(N-1)}\right)$.

$$
\begin{aligned}
\Delta_{L^{I}}(n-1, n-1) & =\frac{3 n-2}{2(N-1)}-c>0 \\
\Delta_{L^{E}}^{3}(n-1, n-1) & =\frac{3 n-1}{2(N-1)}-c>0 \\
\Delta_{H^{E}}^{3}(n-1, n-1) & =\frac{n+1}{2(N-1)}-c \gtrless 0 \\
\Delta_{H^{I}}(n-1, n-1) & =\frac{n+2}{2(N-1)}-c \gtrless 0 .
\end{aligned}
$$

The $H^{E}$ who has the choice will only adopt $D$ if $c<\frac{n+1}{2(N-1)}$. In this case, $D$ will be played by the whole population: all $H^{I}$ and $L^{I}$ have a positive decision term such that $y_{H}$ and $y_{L}$ decline by one each period. Also the last two agents confronted with $D, H^{E}$ and $L^{E}$ imitate $D$, because

$$
\begin{aligned}
\Delta_{H^{E}}^{2}(1,1) & =\frac{3 n-1}{2(N-1)}-c>0 \\
\Delta_{L^{E}}^{2}(1,1) & =\frac{n+1}{2(N-1)}-c>0 .
\end{aligned}
$$

So for $\frac{n}{2(N-1)}<c<\frac{n+1}{2(N-1)}$, the absorbing state is $\vec{D} \vec{D}$.

It is clear that for a slightly higher $c, c \in\left(\frac{n+1}{2(N-1)}, \frac{n+2}{2(N-1)}\right), D$ spreads too in both populations, but once all agents but two play $D, L^{E}$ will not adopt it, according to (31). Because of

$$
\Delta_{L^{I}}(0,1)=\frac{n}{2(N-1)}-c<0,
$$

$C$ will be imitated by the $L^{N E}$ next to $L^{E}$ in the following period, in which case $L^{E}$ switches to $D$ (the decision term for $L^{E}$ now is $\Delta_{L^{E}}^{3}$, which is positive for the $c$ under consideration). All $L$-players but the edge players adopt $C$ then. The edge players' decision term $\Delta_{L^{E}}^{2}$ cannot be negative for the $c$ under consideration and thus the $H$-types are cut off the strategy $C$. For the interval $\frac{n+1}{2(N-1)}<c<\frac{n+2}{2(N-1)}$ we thus find the absorbing state $\vec{D} \vec{C}^{*}$.

Rising $c$ again, $c \in\left(\frac{n+2}{2(N-1)}, \frac{2 n-\frac{1}{2}}{2(N-1)}\right)$, in period $t=4$ none of the $H$-players will imitate $D$ and it will spread in group $L$. Once $y_{L}$ is small enough, group $H$ will adopt strategy $D$. The absorbing state here is $\vec{D} \vec{D}$.

(c) If $\left(\frac{2 n-\frac{1}{2}}{2(N-1)}, 1\right)$, we have $\Delta_{H^{E}}^{2}(n-1, n)<0$ and $\Delta_{H^{I}}(n-1, n)$ respectively. So all players give up $D$ in the second period and the absorbing state is $\vec{C} \vec{C}$. 
(iii)

If an $H^{E}$ introduces $D$, an $L^{E}$ and an $H^{I}$ and the introducing $H^{E}$ can decide to play $D$ or not in the second period. The respective decision terms are

$$
\begin{aligned}
\Delta_{L^{E}}^{4}(n-1, n) & =\frac{3 n-1}{2(N-1)} \\
\Delta_{H^{E}}^{2}(n-1, n) & =\frac{2 n-\frac{1}{2}}{2(N-1)} \\
\Delta_{H^{I}}(n-1, n) & =\frac{n}{2(N-1)} .
\end{aligned}
$$

It is convenient to look at the cost intervals $\left(0, \frac{n}{2(N-1)}\right),\left(\frac{n}{2(N-1)}, \frac{2 n-\frac{1}{2}}{2(N-1)}\right)$, $\left(\frac{2 n-\frac{1}{2}}{2(N-1)}, \frac{3 n-1}{2(N-1)}\right)$, and $\left(\frac{3 n-1}{2(N-1)}, 1\right)$ separately.

(a) If $0<c<\frac{n}{2(N-1)}$, the absorbing state is $\vec{C} \vec{C}$ because all decision terms are positive.

(b) If $\frac{n}{2(N-1)}<c<\frac{2 n-\frac{1}{2}}{2(N-1)}$ then $\Delta_{H^{E}}^{2}(n-1, n)>0$ and $\Delta_{L^{E}}^{4}(n-1, n)>0$, and $\Delta_{H^{I}}(n-1, n)<0$. So in the second period $H^{E}$ and $L^{E}$ only play $D$. This situation is what we have analyzed in Part (ii), subsection (b) of this proof. We can therefore take over these results and conclude the similar dynamics for this range of the costs.

(c) If $\frac{2 n-\frac{1}{2}}{2(N-1)}<c<\frac{3 n-1}{2(N-1)}$ ) only $L^{E}$ plays $D$ in the second period. All $L^{I}$ adopt $D$ in the following because $\Delta_{L^{I}}$ is always negative for the given cost interval. The spread of strategy $D$ among the $L$-types will not affect the $H$-types strategy choice, they keep playing $C$ because $\Delta_{H^{E}}^{4}<0$ for the cost interval under consideration. As discussed before, the $L^{E}$ either cycle between $C$ and $D$ or play $D$, the absorbing state for this cost interval is $\vec{C} \vec{D}^{*}$.

(d) If $\frac{3 n-1}{2(N-1)}<c<1$, the strategy $D$ becomes extinct and the absorbing state is $\vec{C} \vec{C}$, because all decision terms are negative.

\section{Proof of Proposition 6}

We first assume that an $H$-type plays $D$ in period $t=1$. If $\Delta_{H^{E E}}^{4}>0$ he will keep playing $D$ and his two neighbors adopt $D$ if $\Delta_{L^{E E}}^{2}>0$. We have $\Delta_{H^{E E}}^{4}(n-1, n)>\Delta_{L^{E E}}^{2}(n-1, n)$ and so $D$ becomes extinct if $c>\frac{3 n-1}{2(N-1)}$. For $\frac{2 n-\frac{1}{2}}{2(N-1)}<c<\frac{3 n-1}{2(N-1)}$ the equilibrium is the initial strategy distribution, because the neighbors of the inventor do not adopt $D$, but the $H^{E E}$ keeps playing it. 
We focus on $c<\frac{2 n-\frac{1}{2}}{2(N-1)}$ now. As just seen, in $t=2$ only the innovator $H^{E E}$ and his two neighbors play $D$. Four players can choose between $C$ and $D$ now, two $H^{E E}$ (playing $C$ ) that have the decision term $\Delta_{H E E}^{2}$ and two $L^{E E}$ (playing $D$ ) that have the decision term $\Delta_{L^{E E}}^{3}$. Since $\Delta_{H^{E E}}^{2}(n-1, n-2)>\Delta_{L^{E E}}^{3}(n-1, n-2)$ and $\Delta_{H^{E E}}^{2}(n-1, n-2)>0$, there are two cases to be distinguished $t=3$ : either all of them adopt $D$, or the two $H^{E E}$ adopt $D$ and the two $L^{E E}$ do not. The two cases will actually lead to the same absorbing state: the two $L^{E E}$ will have the decision term $\Delta_{L^{E E}}^{1}\left(y_{H}, y_{L}\right)$ in period $t=4$ which is greater than $\Delta_{H^{E E}}^{2}\left(y_{H}, y_{L}\right)$ for all $y_{H}$ and $y_{L}$, so they will adopt $D$ then. Thus, this second case does not influence the absorbing state. It is clear that if the two $H^{E E}$ choose to play $D$, the $L^{E E}$ will do the same one period later. We neglect it from now on.

There are only two different kind of strategy states in this dynamics. The first one is of the kind of period $t=3$ : two $H^{E E}$ with decision term $\Delta_{H^{E E}}^{2}$ and two $L^{E E}$ with $\Delta_{L^{E E}}^{3}$ choose between $D$ and $C$. We call this state $L$-dominated because more $L$-types than $H$-types play $D$ (to be precise: $y_{L}-y_{H}=1$ ). Picture period $t=3$ to get the intuition. In $t=3$, three agents play $D$, these are located as follows:

$$
\underbrace{\ldots H L H L H}_{C} \underbrace{L H L}_{D} \underbrace{H L H L H L \ldots}_{C} .
$$

The second kind of strategy state is of the kind of period $t=4$ : two $L^{E E}$ with decision term $\Delta_{L^{E E}}^{2}$ and two $H^{E E}$ with decision term $\Delta_{H^{E E}}^{3}$ have to decide between the two strategies. We call this state $H$-dominated because more $H$ types than $L$-types play $D\left(y_{H}-y_{L}=1\right)$.

$$
\underbrace{\ldots H L H L}_{C} \underbrace{H L H L H}_{D} \underbrace{L H L H L \ldots}_{C} .
$$

Note that for any positive integer $x, \Delta_{L^{E E}}^{2}(x-2, x-1)$ as well as $\Delta_{H^{E E}}^{3}(x-2, x-1)$ are larger than $\Delta_{H E E}^{2}(x, x-1)$. So if we have reached an $H$-dominated state, we will also reach the $L$-dominated state that features two more $L$-types playing $D$.

We conclude that the absorbing state is reached when $\Delta_{H^{E E}}^{2}$ is negative $\left(\Delta_{H^{E E}}^{2}\right.$ depends positively on $y_{H}$ ). So only an $L$-dominated state can be an absorbing state. The interpretation is that it will always be an $H$-type that stops the spread of $D$. An $H$-type will at one point halt the spread of $D$ and act as a blocker to the $L$-types playing $C$ who would adopt $D$ if they would observe $D$. The higher $c$ 
is, the earlier the spread of $D$ is halted, that means, the less agents use $D$ in the absorbing state. We conclude that there exist many different absorbing states, depending on population size $N$ and the $\operatorname{costs} c$.

We calculate the number of agents playing $C$ in the absorbing state. Since the absorbing state is $L$-dominated, we can substitute $y_{L}$ with $y_{H}-1$.

$$
\begin{aligned}
\Delta_{H^{E E}}^{2} & =\frac{n+y_{H}+\frac{1}{2}}{2(N-1)}-c<0 \\
& \rightarrow y_{H}<2 c(N-1)-n-\frac{1}{2} .
\end{aligned}
$$

The solution $y_{H}$ to this inequality must be an odd (even) number if $n$ is even (odd). This comes from the fact that whenever $H^{E E}$ have the decision term $\Delta_{H^{E E}}^{2}$, there is an odd number of $H$-types playing $D$. This is because $y_{H}$ decreases by steps of two.

If an $L$-type agent innovates $D$ in the first period, the same arguments apply. 


\section{References}

Alos-Ferrer, C., Ania, A. B., and Schenk-Hoppe, K. R. (2000). An Evolutionary Model of Bertrand Oligopoly. Games and Economic Behavior 33 (1), 1-19.

Berentsen, A. and Y. Lengwiler (2004). Fraudulent Accounting and Other Doping Games. Journal of Institutional and Theoretical Economics, forthcoming.

Berninghaus, S., Güth, W., and H. Kliemt (2003). From Teleology to Evolution. Journal of Evolutionary Economics 13 (4), 385-410.

Ellison, G. (1993). Learning, Local Interaction, and Coordination. Econometrica, 61 (5), 1047-1071.

Ellison, G., and D. Fudenberg (1995). Word-of-Mouth Communication and Social Learning. Quarterly Journal of Economics 110(1), 93-125.

Eshel, I., L. Samuelson, and A. Shaked (1998). Altruists, Egoists, and Hooligans in a Local Interaction Model. American Economic Review 88 (1), 157-179.

Kandori, M., G. J. Mailath, and R. Rob (1993). Learning, Mutation, and Long Run Equilibria in Games. Econometrica 61 (1), 29-56.

Kemeny, J. G., and J. L. Snell (1960). Finite Markov Chains. D. Van Nostrand. Vega-Redondo, F., (1997). The Evolution of Walrasian Behavior. Econometrica, 65 (2), 375-384.

Weibull, J. W. (1995), Evolutionary Game Theory. The MIT Press. 